International Journal of Modern Physics C

Vol. 17, No. 5 (2006) 693-710

(C) World Scientific Publishing Company

\title{
MODELLING TRAFFIC FLOW AT MULTI-LANE URBAN ROUNDABOUTS
}

\author{
RUILI WANG* \\ Institute of information Sciences and Technology \\ Massey University, Palmerston North, New Zealand \\ r.wang@massey.ac.nz \\ H. J. RUSKIN \\ School of Computing, Dublin City University \\ Dublin 9, Ireland \\ hruskin@computing.dcu.ie
}

Received 27 May 2005

Revised 2 September 2005

\begin{abstract}
This paper proposes Multi-stream Minimum Acceptable Space (MMAS) Cellular Automata (CA) models to study unsignalised multi-lane (two- or three-lane) urban roundabouts. Through detailed space considerations, using Cellular Automata (CA) and the Multi-stream Minimum Acceptable Space method, heterogeneity and inconsistency of driver behavior and interactions in cross traffic at entrances of roundabouts are simulated by incorporation of four different categories of driver behavior (i.e., conservative, moderate, urgent and radical), together with reassignment of categories with given probabilities at each time step. The method is able to reproduce many features of urban traffic, for which gap-acceptance models are not robust. Multi-lane roundabout models, in particular for two-lane roundabouts, are developed with different vehicle lane-allocation patterns. Various properties of multi-lane roundabout operations have been explored including throughput, turning rates, critical arrival rates and congestion. The operations of two- and three-lane roundabouts are compared in terms of throughputs. Vehicle movements in this paper relate to left-side driving, such as found in Ireland, New Zealand and the UK. However, results are generally applicable to the countries where the give-way rule is applied.
\end{abstract}

Keywords: Traffic flow modelling; multi-lane roundabouts; cellular automata; Multistream Minimum Acceptable Space.

\section{Introduction}

Modelling multi-stream traffic flow is a challenging task. In particular, the heterogeneous nature of human behavior, the random interactions between drivers, the

* Corresponding author. 
highly nonlinear dynamics and the large dimensions of the system under investigation combine to create considerable complexity.

Multi-lane (two- or three-lane) roundabouts are a next-step urban traffic alternative in situations where single-lane roundabouts prove inadequate. Unsignalised two-lane roundabouts are commonly used in Ireland and the UK, whereas three-lane roundabouts are widely used in New Zealand. "Unsignalised" means that cross traffic at the intersections or the entrances of unsignalised roundabouts is not controlled by any traffic light, but by the give-way rule (the priority rule). According to the give-way rule, vehicles from minor streets give way to vehicles from the major streets of an intersection, while vehicles from the entry roads of a roundabout give way to those vehicles already on the roundabout (also called circulating vehicles). The give-way rule is common to a number of countries, such as Germany, Ireland, New Zealand and the UK, but is not universal to all.

Gap-acceptance models have received much attention in modelling traffic flow at the entrances of roundabouts and intersections. ${ }^{1}$ Obviously, treating the entrances of roundabouts similarly to those of intersections does not reveal the operational characteristics of roundabouts. Limitations of gap-acceptance models are discussed in Sec. 2 in general, but one major drawback is that these models lack scalability. In other words, they can be used for an individual entrance, but are more difficult to apply and interpret in modelling a roundabout as a whole or modelling a traffic network.

Cellular automata $(\mathrm{CA})$ models provide an efficient alternative way to model traffic flow for highway and urban networks. ${ }^{2-8} \mathrm{CA}$ models have been widely used to simulate traffic flow with a single direction of movement, such as these found on highways, one-way streets or ramps. ${ }^{9}$ Some research has been conducted on bi-directional traffic flow. ${ }^{10,11}$ However, limited research has been conducted on cross traffic, in particular, unsignalised cross traffic flow, which is one of the most important features in urban traffic networks. ${ }^{12}$

Early research on urban networks was done by Biham et al. ${ }^{7}$ This model, often called the BML model, uses a simple two-dimensional square lattice to simulate cross traffic, but does not consider the randomness inherent in traffic behavior. An exception to this is the initial condition that seeds vehicles to streets randomly. ${ }^{13}$

The original BML models aimed to simulate one-way traffic, and were subsequently further developed to allow two-way traffic on all streets. ${ }^{14}$ The model proposed in Ref. 14 implicitly takes into account the possibility of formation of queues. $^{8}$ Similar to other models based on BML, there is, however, the problem that no details of vehicle motion and interaction are considered.

A CA rotary was initially proposed for unsignalised intersections in Refs. 3 and 15. All entry roads are "connected" on the rotary. The car "on the rotary" has priority over any new entry. A major issue is that there is no differentiation between the major and minor entry roads of the intersection and all vehicles have equal priority to move into the "rotary" (i.e., the intersection), which compromises the usual give-way rule governing Two-Way-Stop-Controlled (TWSC) intersections. In other 
words, the model does not reflect the interactions between drivers at unsignalised intersections. A further CA model variant for intersections is described in Ref. 16, which uses a simplified approach to account for the give-way rule. If a vehicle from a major road approaches, then vehicles from the minor roads just wait, regardless of their proposed directions of movement.

A more realistic CA model was first proposed by Ruskin and Wang, ${ }^{5}$ which simulates the cross traffic at unsignalised intersections, reflects the give-way rule appropriately and describes the details of vehicle movements and interactions from different entry roads. The model was based on the Minimum Acceptable sPace (MAP) method proposed by Wang and Ruskin. ${ }^{6}$ MAP can be designed to describe both heterogeneous and inconsistent driver behavior, as well as random interaction between individual vehicles in cross traffic flow, independent of headway distribution considerations. ${ }^{1}$ As such, the MAP method can be applied to most features of traffic flow ${ }^{5}$ and has been used for the single-lane roundabout and intersection..$^{5,6}$

Note that CA models have been used before in simulating heterogeneous and inconsistent driver behavior, but for progression along a straight road, not in cross traffic manoeuvres. For example, the updated Nagel and Schreckenberg model ${ }^{17}$ used randomisation to simulate three different properties of human driving in one computational operation. The three different properties are fluctuation at maximum speed, over reaction on breaking, and retard (noisy acceleration).

Wang and Ruskin proposed the first CA model, using the Minimum Acceptable sPace (MAP) method, to simulated cross traffic flow at unsignalised single-lane roundabouts. ${ }^{6}$ In this paper we develop a Multi-stream Minimum Acceptable Space (MMAS) CA model, based on the MAP method,,$^{5,6}$ to simulate the cross traffic at unsignalised multi-lane roundabouts.

Performance measurements for roundabouts include throughput (the maximum number of vehicles that can navigate a roundabout) and capacity (the maximum number of vehicles that can pass through a roundabout from a given road), as well as queue lengths, waiting time, passage time and so on. All the performance measures mentioned can be readily obtained by Multi-stream Minimum Acceptable Space (MMAS) Cellular Automata (CA) models, as above. In this paper, however, we mainly study throughputs, which suffice to give an integrated picture of roundabout performance.

Finally, CA models are currently used by a research group in China ${ }^{18}$ to simulate two-lane "plane traffic circles" (which are similar to roundabouts). In their model, the standard give-way rules (governing vehicle entrance to a roundabout) are not applied. Instead, priority is given to the vehicle which arrives first at the conflicting point (where cross traffic flows meet/merge) based on their current speeds. This rule is applied generally in China, in terms of governing the self-organising process of unsignalised traffic flow. Clearly, driver behavior is, to some extent, culture-based.

This paper is organised as follows. In Sec. 2, the limitations of gap-acceptance models have been analysed. In Sec. 3, lane-allocation patterns and the MMAS 
method are introduced. In Sec. 4, simulation results are given and some findings are described. Finally, a summary is given in Sec. 5 .

\section{Background}

A common deficiency of all previous models that study cross traffic flow is the assumption that drivers are consistent and homogeneous. ${ }^{1}$ In reality, both inconsistent and heterogeneous behavior is endemic. This realisation provides a principal motivation for much of the work described here.

Gap-acceptance models have long been used in modelling cross traffic at the entrance of roundabouts and intersections and have primarily been used for singlelane roundabouts or intersections.

Gap-acceptance models assume that a driver will enter the intersection when a safe opportunity or gap occurs in the traffic. Gaps are measured in time and correspond to headway (defined as distance divided by speed). Critical gap and follow-up time are the two key parameters (where critical gap is defined as the minimum time interval required for one minor-stream vehicle to enter the intersection). Gap-acceptance models are, however, unrealistic in assuming consistent driver behavior. ${ }^{19,20}$ A consistent driver would be expected to behave in the same way in all similar situations, while in a homogenous population, all drivers have the same critical gap and are expected to behave uniformly. ${ }^{21}$ More realistically, driver type may differ and the critical gap for a particular driver should be represented by a stochastic distribution, ${ }^{22}$ and this should be reflected in the simulation.

In gap-acceptance models, estimation of the critical gap has attracted much attention, with use of a mean critical gap also proposed. ${ }^{23-25}$ Maximum likelihood estimation of the mean critical gap has gained wide acceptance, ${ }^{1,24-27}$ but the basic assumption remains the same, namely that all drivers are consistent.

Investigation of the factors affecting critical gap and follow-up time concluded that drivers use a shorter critical gap at higher flow and delay conditions. ${ }^{28}$ Many other factors also operate, ${ }^{28-30}$ however, so that a critical value obtained for any given situation is unlikely to be generally applicable.

Further, at roundabouts in an urban network, adjacent intersections with traffic lights will have grouped the vehicles into a queue (or queues) during the red signal phases and platoons will thus be present (e.g., a filtering effect). The filtering of traffic flow by traffic signals has a significant impact on capacity and performance. ${ }^{31}$ In particular, the gap-acceptance model can be applied only when no platoon is present. ${ }^{32}$ Otherwise, no minor-stream vehicle can enter the intersection or roundabout, as the mean headway within a platoon is supposed to be less than the critical gap. If traffic signal cycles are known and co-ordinated, the platoon pattern may be predictable. If it is not predictable, traditional gap-acceptance cannot readily be applied ${ }^{32}$ and furthermore, does not specifically allow for modelling directional flow. ${ }^{28}$ 
Priority sharing is another phenomenon that gap-acceptance models fail to take into account. This occurs at the entrance to a roundabout where circulating vehicles may deliberately give way to entering vehicles. ${ }^{33}$ This appears to lead both to reduction in the critical gap and in the average follow-on time for entering vehicles, which causes inaccuracy in the capacity model, based on the gap-acceptance criteria. Troutbeck and Kako ${ }^{34}$ have tried to overcome this by adding an additional factor " $C$ " in the capacity formula to justify the priority sharing effects. This $C$ value ranges from 0 to 1 and depends on the headway distribution. Although this modification can improve the accuracy of previous gap-acceptance models, it obviously provides little help in analysing the cross traffic operation unless priority sharing can be directly related to the headway distribution.

Research on multi-stream cross traffic flow has focused on the estimation of critical gaps in multi-major-streams. ${ }^{26,35,36}$ The EM algorithm ${ }^{37}$ and edge distribution $^{26,35}$ have been used respectively to estimate the critical gaps in $\mathrm{T}$ junctions (with two major streams), ${ }^{35}$ and also to observe rejected and accepted gaps in a major lane (when gaps in other major lanes are so large that these could not influence drivers on a minor stream). ${ }^{26,35}$

These authors (see Refs. 26, 35 and 36) estimated different critical gaps for different streams, but results for intersections and roundabouts were contradictory. Questions of impedance of the vehicles in minor and major streams were also variously considered, but findings on the number of opportunities presented for vehicles to move onto the roundabout are not well substantiated. Field indications are that position delay checks should be taken into account. ${ }^{12}$

The Minimum Acceptable sPace (MAP) method was first proposed in Ref. 6, using analogous but more flexible methodology to that of gap-acceptance (e.g., spatial and temporal details of vehicle interactions can be described using MAP). This not only facilitates understanding of the interaction between drivers, but can also be applied to situations for which headway distributions are insufficient to describe traffic flow (e.g., traffic flow filtered by traffic signals). We use a multi-CA ring to extend previous work on the single-lane roundabout ${ }^{6}$ to the multi-lane case.

Note that the notion of gap-acceptance has been used in modelling two-lane traffic, with overtaking allowed if the gap on the other lane is large enough (e.g., in Ref. 38). However, these models again are for single directional multi-lane traffic flow, which is different from traditonal gap-acceptance models and the developed in this paper.

\section{Methodology}

Vehicles on single- and multi-lane roundabouts are assumed to observe the same give-way rule (the priority rule), as follows: entering vehicles must give way to the vehicles already on a roundabout. Also, for multi-lane roundabouts, vehicles on the middle and outside/outer lanes must give way to vehicles leaving from the inside/inner lanes. 
Vehicles on entry roads move onto the corresponding lanes of the roundabout, while navigation through a roundabout is subject to the following processes:

(1) Vehicle arrival at start of entrance road (e.g., 100 cells away from the roundabout).

(2) Predetermined destination (before allocation to a lane of the entry road).

(3) Lane allocation (on entry road).

(4) Vehicle movement along entrance roads.

(5) Position delay: vehicles on the left lane (or on the middle lane) of an entrance road may be halted for position delay time (PDT), if view is impeded by the adjacent vehicle, in order to adjust position/check opportunity to enter roundabout.

(6) Entry (interaction between drivers at the entrance and vehicles on the roundabout).

(7) Navigation on the roundabout.

(8) Exit.

In this paper, we focus on the third, sixth and eighth processes identified above, as others are similar to the single-lane case. ${ }^{6}$ It is more realistic to assume that the destination is predetermined and remains unchanged for all vehicles throughout the roundabout manoeuvre. Therefore, all the vehicles in our model will randomly be assigned a destination according to turning rates when entering the system. Their destinations will remain unchanged during their manoeuvres.

\subsection{Lane allocation}

In the lane allocation process, a vehicle chooses which lane it will use to approach a roundabout. The lane allocation process at a two-lane roundabout is similar to that for the major roads of a two-lane Two-Way-Stop-Controlled (TWSC) intersection in Ref. 39. However, criteria for lane allocation differ slightly. For example, the feature that only right-turning (RT) vehicles may use the right lanes is common for two-lane TWSC intersections, but is rare for two-lane roundabouts. We simulate two possible patterns in the real world:

- Left-turning (LT) vehicles using left lane only. Straight-through (ST) and RT vehicles using right lane only, i.e., only LT vehicles use the outer lane of a roundabout (Pattern A)

- LT vehicles using left lane only. RT vehicles using right lane only, and ST vehicles using both lanes (Pattern B)

In the first scenario, the vehicles travelling on the outer lane of the roundabout are LT vehicles only. The advantage of this system is that entry vehicles need to check the space on the inner lane of the roundabout only, since there is no oncoming vehicle from the outer lane. The manoeuvre is a merging process between the vehicles in the circulating flow on the inner lane of the roundabout and vehicles 
on the right lanes of entry roads and no cross interaction occurs. Unfortunately, in reality ST vehicles will take the left lane if the right lane of the entrance road is saturated.

The second scenario is used to give greater flexibility to the ST vehicles. Selection by the driver of a lane might be based, e.g., on perception of waiting time or similarly. As ST vehicles can use the outer lane, some passing interaction occurs when vehicles on the inner lane of the roundabout are exiting.

The lane allocation process on a three-lane entrance road of a three-lane roundabout is relatively simple as RT vehicles will use only the right lane, LT vehicles the left lane and ST vehicles the middle lane of the entrance road. However, the interaction at the entrance of the roundabout is more complicated than that for a single- or two-lane roundabout.

\subsection{Minimum Acceptable sPace (MAP)}

We briefly outline the MAP method (see Refs. 5 and 6) before describing MMAS models. In the MAP method, driver behavior is categorised into four groups: radical, urgent, moderate and conservative. We use a single-lane roundabout to illustrate the MAP method in Fig. 1. If a driver accepts a 3-cell space (between circulating vehicles) as the MAP and enters the roundabout, behavior is designated as moderate. Radical behavior requires one cell space. The driver will take any space on the intersection without any consideration of safety. In consequence, this vehicle may delay the oncoming vehicle on the roundabout for two time steps (corresponding to two seconds in our model) and may generate gridlock $^{6}$ (see Sec. 4). A 2-cell space corresponds to urgent behavior, which may be the result of such things as misjudgement, over confidence in vehicle acceleration, bad driving habits, urgency of travel or the phenomenon of priority sharing. The effect is to delay an oncoming vehicle on the roundabout. Conservative behavior corresponds to MAP $\geq 4$ cells.

MAP and MMAS are a combination of both spatial and temporal conditions. For example, MAP is determined by the number of cells needed to represent driver behavior of the various types, when entering a single-lane roundabout. In the model

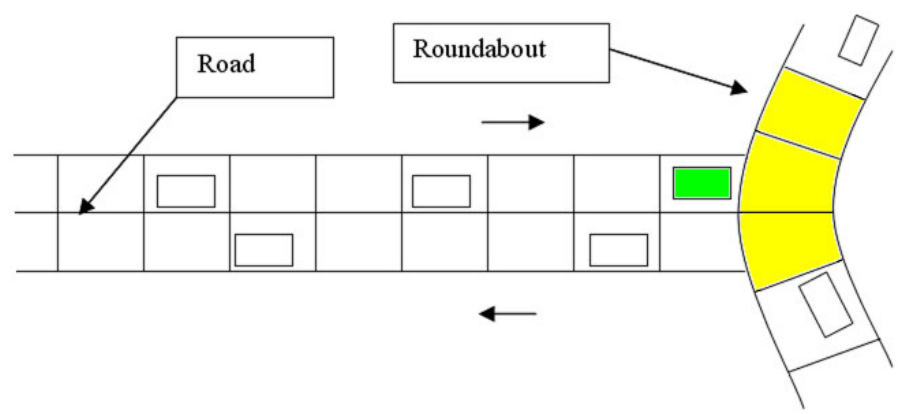

Fig. 1. A road and its entrance to a roundabout. 
a vehicle can move forward one cell in each time step on a roundabout. Therefore, the number of cells of MAP correspond to the headway between the two circulating vehicles on a roundabout. On the other hand, the space for a vehicle to enter a roundabout must have clearance of specific cells for entry, otherwise the entering vehicle must wait (see Fig. 1 for MAP and Figs. 2-4 for MAAS) to join different roundabout lanes. Therefore, MAP and MMAS describe both temporal and spatial behavior of drivers in cross traffic.

\subsection{Interaction at entrances of multi-lane roundabouts}

In Ref. 6, a multi-state CA ring is developed in order to characterise vehicle destinations. In other words, the states of a vehicle on a roundabout depend on the distance (the number of cells) to its destination. In this paper, multi-state two- and three-CA rings are used to simulate two- or three-lane roundabouts (two or three cellular automata rings with the same centre but different diameters). All rings have the same number of cells, and vehicles can move ahead one cell in each time step when navigating the roundabout. In other words, we assume that vehicles in all lanes traverse the same number of radians in the same period of time. This is permitted by the assumption of an adjustment in the speed of the vehicles on a roundabout with different radius. The shorter the radius, the lower the speed at which that vehicle can move. The state of a vehicle thus depends on the distance (the number of cells) to its destination and which lane it is in.

In this paper, we use vehicles in the right lane of entrances to two- and threelane roundabouts to show the interactions which occur. In order to simplify the representation, the shape of the arc of the roundabout with entry road can be stretched to resemble Figs. 2 and 3, which look like a T-intersection. The paths of vehicles in the entry road are shown in Figs. 2(d) and 3(d), while the paths of vehicles exiting from the roundabout are shown in Figs. 2(c) and 3(c). When the
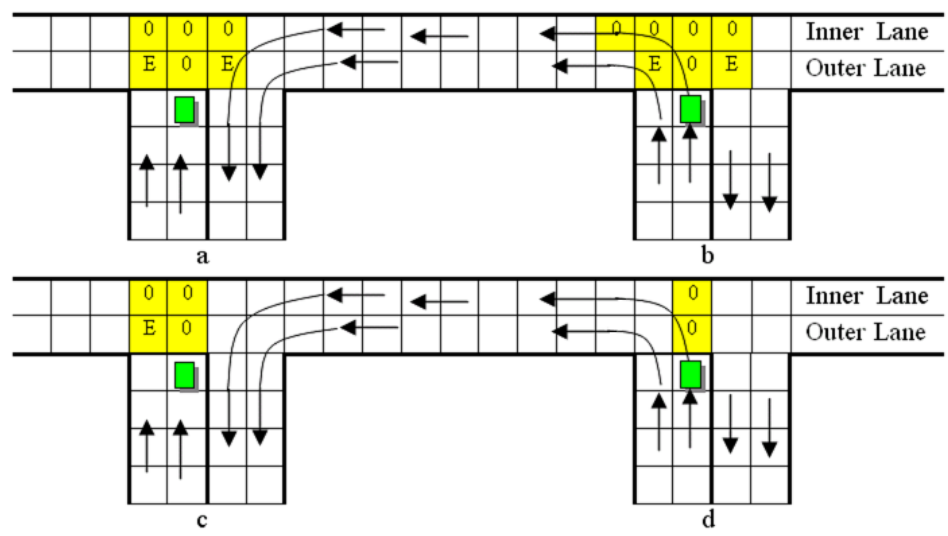

Fig. 2. Vehicle on the right lane of the two-lane entrance road with behavior of (a) moderate, (b) conservative, (c) urgent and (d) radical. 

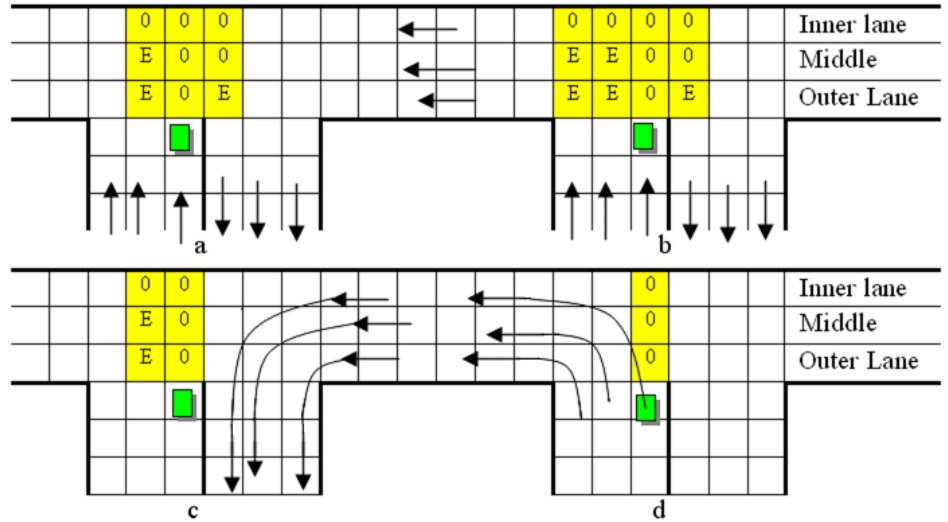

Fig. 3. Vehicle in the right lane of the three-lane entrance road with behavior of (a) moderate, (b) conservative, (c) urgent and (d) radical.

vehicle in the middle or right lane of the entance road needs to change lane from the outer lane to the middle and/or inner lane on the roundabout, it crosses the two cells diagonally. Likewise, this is true for the vehicles coming out from the inner lane (and/or the middle lane) to the outer lane (see the curved arrows). In other words, when a vehicle changes lane on the roundabout, it also moves one cell ahead at the same time.

Similar to the MAP method in Ref. 6, we use figures to explain our MMAS model and the conditions that are required by vehicles from entrance roads. Driver behavior is categorised into four groups: conservative, moderate, urgent and radical, with associated probabilities. ${ }^{6}$

For a two-lane roundabout, the required conditions for the target vehicle (shaded) on the right lane of the entry road in this time step are indicated by the spaces required (marked cells) in Figs. 2(a) and 2(b), based on two different types of driver behavior.

Figure 3 shows vehicles entering from the right lane of the entrance road of a three-lane roundabout. In this time step the required conditions for the target vehicle (shaded rectangle) on the left lane of the entrance road, are indicated by the spaces required (marked cells) in Figs. 3(a)-3(d), based on four different driver behavior assumptions described. Figure 4 shows vehicles entering from the middle and right lane of the entrance road of a three-lane roundabout.

The requirement for each cell is indicated by " 0 " or " $E$ ", where " 0 " means that the cell must be vacant and " $E$ " means that the cell is either vacant or occupied by a non-circulating vehicle. A non-circulating vehicle is one either just entering the roundabout from an entrance road or going to leave the roundabout in the next time step. All space requirements are indicated cell by cell (with the same notation "0" or "E"). Theoretically, the left lanes of entrance roads and the outer lanes of roundabouts are designed for left-turning vehicles only. Thus, the left-turning 


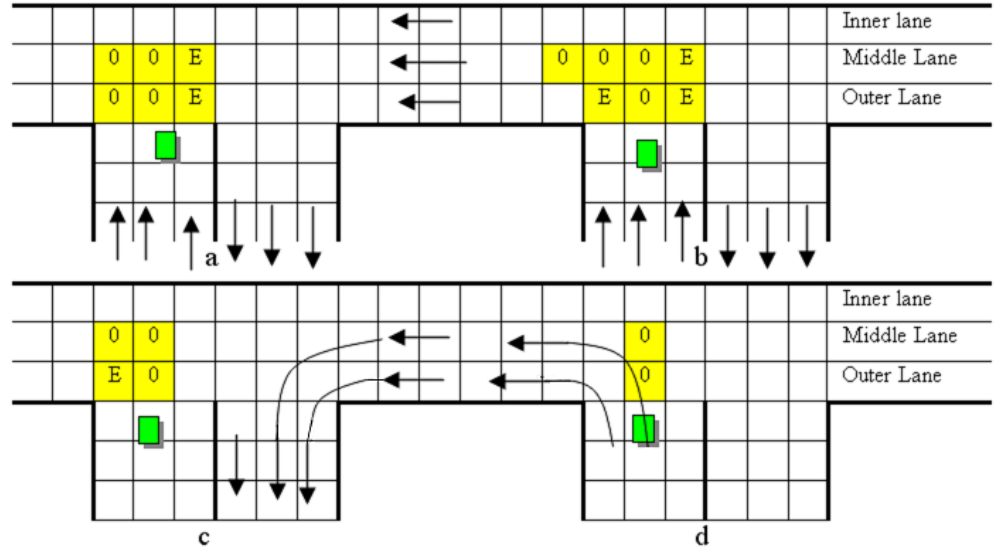

Fig. 4. Vehicle on the right-lane of the three-lane entrance road with (a) moderate, (b) conservative, (c) urgent and (d) radical behavior.

vehicles do not need to check conditions before entering. However, in practice, checking is clearly necessary for safety reasons and is built into the models.

For vehicles entering from the middle and right lanes, we assume that drivers use similar space requirements for each lane that vehicles will traverse. Thus, MAAS covers 3 cells in the outer and middle lanes of the roundabout in both Figs. 3(a) and $4(\mathrm{a})$.

The assumption of similar space requirement for each lane is justified by the argument that drivers' heterogeneous behavior is partially determined by their types and individual characteristics, such as sex, age and driving experience, among others, ${ }^{40}$ and not by their locations in different lanes. Some investigations indicate that age is an important factor in determining not only driver reaction time, but also behavior, ${ }^{40,41}$ although other arguments suggest that a driver who accepts a small gap in one lane is more likely to use a larger gap in the other lane in order to compensate for the risk. ${ }^{42}$

Further suggestions are that two types of interactions are involved, crossing and merging, or that the passing speeds which the entering vehicle may reach to pass the near and far lane are different. Larger gaps in the near lane and smaller gaps in the far lane have been reported in some studies, together with other suggestions as to why drivers vary, but results are not in good agreement overall. ${ }^{26,35,36}$

Our view is that all possibilities strongly reflect the individual driver. A "risktaker" takes the same amount of risk, no matter whether the risk is equally or unequally distributed between the two lanes (in agreement with Ref. 35). On the other hand, a "risk-averse" decision implies equal caution in both lanes. The assumption of equal space requirements in each lane can be seen as a compromise in this case. 


\subsection{Interaction on roundabouts}

Immediately after entering a roundabout, vehicles from the middle and right lane of the entrance roads move from the outer lane into the middle and inner lane respectively. These are assumed to move along the middle lane and inner lane until they arrive at the destinations (exit roads). In other words, they do not change lanes except for entering and exiting. This assumption is supported by the fact that unnecessary lane-changing on roundabouts is not common. ${ }^{12}$

For a three-lane roundabout, exit of the vehicles on the outer and middle lanes is expected to be free flow. Nevertheless, exit of the vehicles on the inner lane may be blocked by vehicles driving on the middle lane. The Give-Way Rate (GWR) is a probability assigned to this random result of driver interactions. The probability ranges from 0 (no driver gives way) to 1 (all drivers give way).

\section{Model Experiments}

In order to study roundabout performance, the following computer experiments have been carried out. The focus here is on results from two-lane roundabouts. In each experiment, the length of each entrance road is 100 cells. If throughput is printed in bold (as in Table 1 for instance), this means that the queue length has reached the length of the road on all entrance roads, i.e. saturated. All experiments are carried out for $3 \times 36000(=3 \times 60 \times 60 \times 10=3 \times 10$ hours $)$ time steps. If an arrival rate $(\mathrm{AR})=0.01$, it is equivalent to $A R=36 \mathrm{vph}$ (vehicles per hour).

In particular, performance is assessed for computer experiments observing (i) throughput versus arrival rates, (ii) throughput versus turning rates, (iii) Position Delay Time (PDT) and GWR on the roundabout versus throughput, (iv) driver behavior versus throughput, (v) queue formation for roundabout and individual road, and (vi) individual road performance (i.e., queue lengths). Results from (i), (ii) and (iv) are presented explicitly here. Others are discussed only briefly.

\subsection{Relationship between throughput and arrival rates}

Figure 5 shows throughput variation with arrival rates for Pattern A. Arrival rates of three roads are taken to be the same (i.e., $A R_{2}=A R_{3}=A R_{4}$ ) and are allowed

Table 1. Driver behavior versus throughput for Pattern A.

\begin{tabular}{lcccccr}
\hline \multirow{2}{*}{$\begin{array}{c}\text { Driver } \\
\text { Behavior }\end{array}$} & \multicolumn{5}{c}{$\left(A R_{1}=A R_{2}=A R_{3}=A R_{4}\right)$} \\
\cline { 2 - 7 } & 0.20 & 0.25 & 0.30 & 0.35 & 0.40 & 0.45 \\
\hline Pco $=1$ & 2878 & $\mathbf{2 9 8 8}$ & $\mathbf{3 1 6 8}$ & $\mathbf{3 3 5 6}$ & $\mathbf{3 5 3 2}$ & $\mathbf{3 7 0 5}$ \\
Pmo $=1$ & 2880 & 3583 & $\mathbf{4 1 6 3}$ & $\mathbf{4 3 3 2}$ & $\mathbf{4 5 1 6}$ & $\mathbf{4 7 0 4}$ \\
Pur $=1$ & 2877 & 3598 & $\mathbf{4 1 6 1}$ & $\mathbf{4 3 3 6}$ & $\mathbf{4 5 2 5}$ & $\mathbf{4 7 0 2}$ \\
Pra $=1$ & $\mathbf{7 6 5}$ & $\mathbf{9 5 0}$ & $\mathbf{1 0 7 4}$ & $\mathbf{1 2 5 3}$ & $\mathbf{1 4 4 1}$ & $\mathbf{1 6 4 6}$ \\
\hline
\end{tabular}




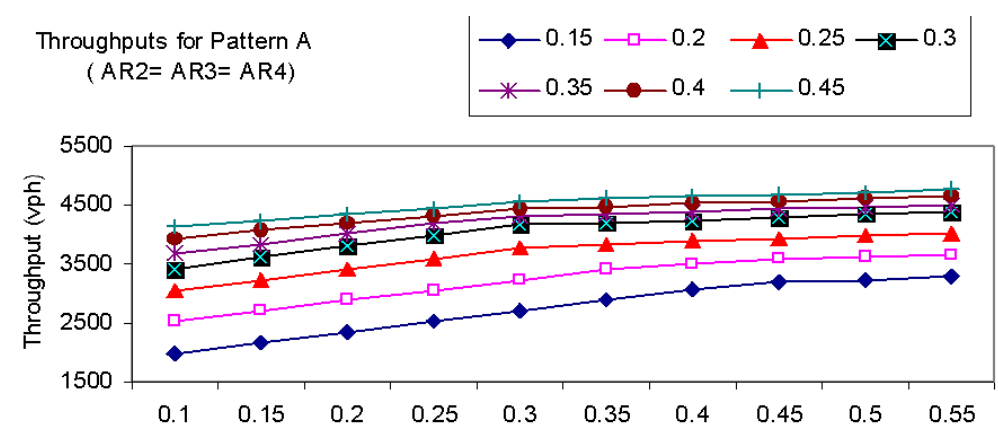

Fig. 5. Throughput versus arrival rates for Pattern A.

to range from 0.15 to 0.45 . Arrival rate of road $1\left(A R_{1}\right)$ also increases from 0.10 to 0.55 .

The above findings for Pattern A can be summarised in the following three expressions. When the arrival rate of the entry road $\geq$ critical arrival rate (CAR), saturation occurs on the entry road. The empirical relationships between $C A R_{1}$ (of road 1) and arrival rates of the other three roads are:

$$
\begin{gathered}
\text { If } A R_{i} \leq 0.05, \quad \text { then } C A R_{1}=0.55, \\
\text { If } 0.05<A R_{i} \leq 0.30, \quad \text { then } C A R_{1}=0.60-A R_{i}, \\
\text { If } A R_{i}>0.3, \text { then } C A R_{1}=0.3,
\end{gathered}
$$

where $i$ (subscript) $=2,3$ or 4 . The above formulae also can be expressed in terms of vph (vehicles per hour):

$$
\begin{gathered}
\text { If } A R_{i} \leq 180 \mathrm{vph}, \quad \text { then } C A R_{1}=1980 \mathrm{vph}, \\
\text { If } 180<A R_{i} \leq 1080 \mathrm{vph}, \quad \text { then } C A R_{1}=2160-A R_{i}, \\
\text { If } A R_{i} \geq 1080 \mathrm{vph}, \quad \text { then } C A R_{1}=1080 \mathrm{vph},
\end{gathered}
$$

where $i=2,3$ or 4 .

Throughput of the Pattern A two-lane roundabout (Sec. 3.1), continues to increase with arrival rate when all roads are saturated (i.e., arrival rate $\geq C A R$ ). The situation is different from that in single-lane roundabouts in Ref. 6, since Pattern A only allows LT vehicles to use the left lane of the entrance road and traffic on the left lane is always free flow. Therefore, when arrival rates increase, the number of LT vehicles continues to increase. Consequently, throughput also increases.

The findings for Pattern B (Sec. 3.1) can also be summarised in the following expressions. The empirical relationships between $C A R_{1}$ and arrival rates of other three roads (see Fig. 6) are:

$$
\text { If } A R_{i} \leq 0.45 \text {, then } C A R_{1}=0.85-A R_{i},
$$




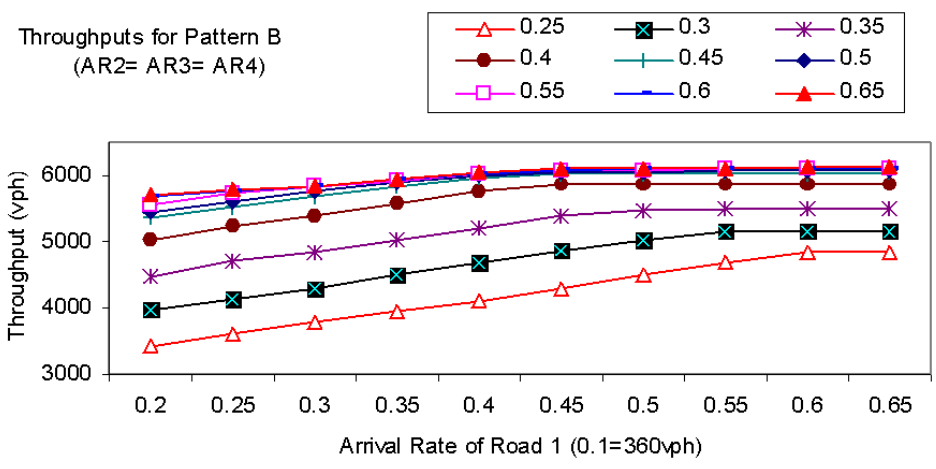

Fig. 6. Throughput versus arrival rates for Pattern B.

If $A R_{i}>0.45$, then $C A R_{1}=0.40$,

where $i$ (subscript) $=2,3$ or 4 .

Comparing the critical arrival rates and throughputs of Patterns A and B, we find that operational performance of Pattern $\mathrm{B}$ is better than Pattern A. We also find that balanced arrival rates (i.e., $A R_{1}=A R_{2}=A R_{3}=A R_{4}$ ) lead to improvement in operational performance of the roundabout for both Pattern A and Pattern B. If we define the effective throughput as the throughput when no entrance road is saturated, the maximum effective throughput that we find is $3665 \mathrm{vph}$ for Pattern A when $A R_{1}=A R_{2}=A R_{3}=A R_{4}=0.28$ and 5806 vph for Pattern $\mathrm{B}$ when $A R_{1}=A R_{2}=A R_{3}=A R_{4}=0.43$. When arrival rates are not equal, the effective throughput is less than optimal.

For single-lane roundabouts, the relationship between throughput and arrival rates is closely related, since vehicles arriving from any road need to wait for the minimum acceptance space to enter the roundabout. However, for two- or three-lane roundabouts, throughput also depends on turning rates. In particular, it is dependent on left-turning rates as left-turning vehicles on the outer and left lanes are theoretically free flow. For larger proportion of left-turning vehicles, the throughput will be higher. Thus, we focus on the relationship between throughput and straight-ahead and right-turning vehicles. In the following computer experiments, therefore, left-turning rate is assumed to be fixed at 0.25 , i.e., only $25 \%$ of arriving vehicles are expected to turn left.

We find that the critical arrival rate of a three-lane roundabout is approximately the same as the critical arrival rate of a two-lane roundabout with Pattern B (see Expressions (7) and (8)). This implies that three-lane roundabouts will not increase throughput compared to two-lane roundabouts (if all drivers observe the rules and the two-lane roundabouts use Pattern B). This finding contradicts the usual perception that operational performance of three-lane roundabouts is better than that of two-lane roundabouts, and probably reflects the expected free flow 
for LT vehicles in the former. Thus, the performance of a three-lane roundabout is decided by the performance of its middle and inner lane, effectively a reduction to the two-lane case.

\subsection{Relationship between throughput and turning rates}

Figure 7 shows the relationship between throughput and turning rates for a twolane roundabout with Pattern A. The mean ST rate $(\mathrm{STR})$ is fixed $(=0.5)$. The mean right turning rate (RTR) is allowed to increase from 0.05 to 0.45 in increments of 0.05 , while LT rates (LTR) consequently vary from $0.45-0.05$. As the arrival rate increases from $0.15-0.45$, we see that traffic on the entry road (to the roundabout) changes from free flow to saturation. When RTR increases by 0.10 , this gives around a $15 \%$ decrease in throughput when the entrance roads are saturated.

Figure 8 shows the relationship between throughput and right-turning rates (RTR) of Pattern B. When $A R_{1}=A R_{2}=A R_{3}=A R_{4}<0.3$, traffic flows freely and turning rates have no impact on throughput. When all arrival rates are set $=$ 0.3 and right-turning rate is 0.35 , traffic still flows freely. However, if right-turning rates are increased to 0.45 , entrance roads are rapidly saturated and turning rates

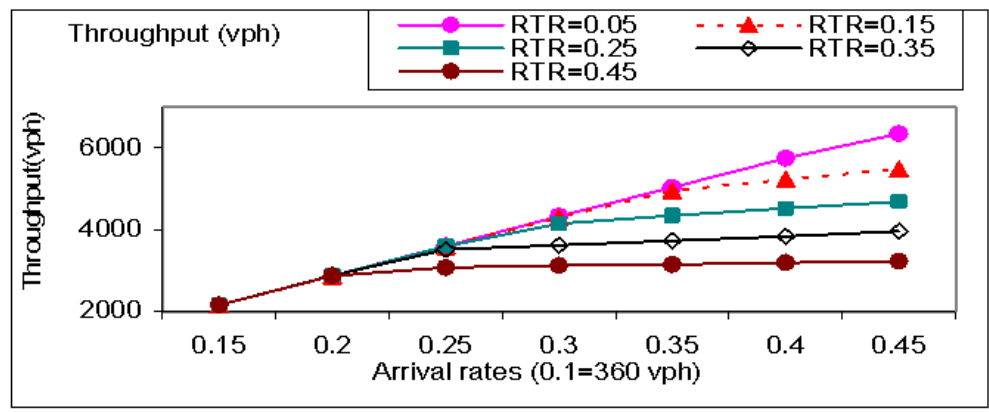

Fig. 7. Throughputs change versus right-turning rate for Pattern A.

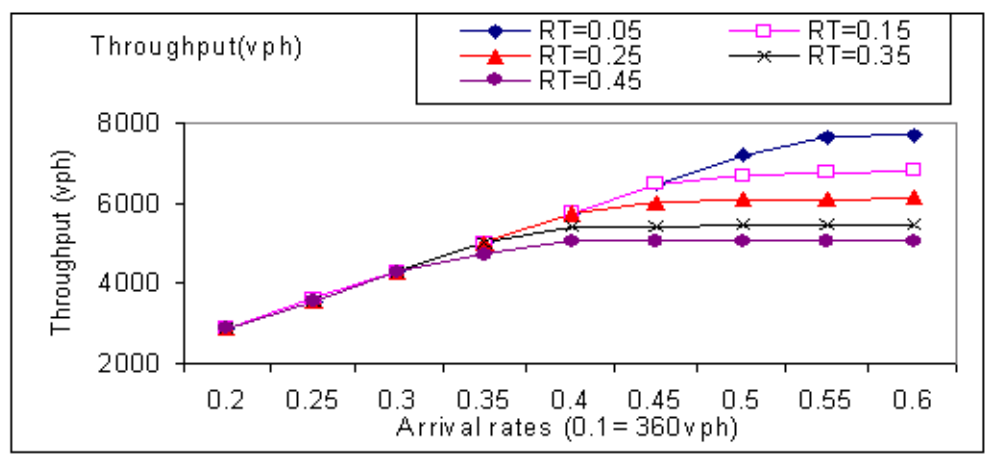

Fig. 8. Throughput versus RTR rate for Pattern B. 
do affect throughput. When $A R_{1}=A R_{2}=A R_{3}=A R_{4}>0.3$, turning rate is again important. In general, when RTR is increased by 0.10 this gives around a $10 \%$ decrease in throughput when entrance roads are saturated. The relationship between right-turning rate (RTR) and CAR can be roughly expressed by the following empirical relationship:

$$
C A R=0.575-0.5 R T R .
$$

\subsection{Driver behavior}

The impact of driver behavior on throughput in Patterns A and B can be shown in the following computer experiments. We assume that the sum of probabilities of conservative $\left(P_{c o}\right)$, moderate $\left(P_{m o}\right)$, urgent $\left(P_{u r}\right)$ and radical $\left(P_{r a}\right)$ behavior is equal to $1 .{ }^{6}$ In this case, and for simplicity, the outcomes for all drivers are of one type are considered in the first instance. These are clearly special situations, which are examined to give some indication of how extremes of driver behavior impact on two-lane roundabout performance. A mixed driver set is of course also possible and easily tested with our models.

Tables 1 and 2 show the results for Patterns $\mathrm{A}$ and $\mathrm{B}$, with equal arrival rates in each column. For all $A R=0.20$ (i.e., $A R_{1}=A R_{2}=A R_{3}=A R_{4}=0.20$ ) for Pattern A and all $A R=0.30$ for Pattern $\mathrm{B}$ in column 1, all throughputs are the same except for all drivers radical (i.e., $P_{r a}=1$ ). When $P_{c o}=1$ and all $A R \geq 0.25$ for Pattern $\mathrm{A}$ and all $A R \geq 0.35$ for Pattern $\mathrm{B}$, throughput reaches the maximum and a saturated situation occurs on entrance roads, while traffic flow on the roundabout remains free flow at all times. When $P_{m o}=1$ or $P_{u r}=$ 1, throughputs for Pattern A are similar (though different for Pattern B), but larger than those for $P_{c o}=1$. Traffic flow on the roundabout again remains free at all times. When $P_{r a}=1$, all $A R>0.20$ for Pattern $\mathrm{A}$ and all $A R>0.30$ for Pattern $B$, throughputs are reduced compared to the previous case, as congestion forms on the roundabout itself. Similar results are also found with other turning rates.

Note that results for three-lane roundabouts are broadly similar to those found here for Pattern B.

Table 2. Driver behavior versus throughput for Pattern B.

\begin{tabular}{lccccccc}
\hline \multirow{2}{*}{$\begin{array}{c}\text { Driver } \\
\text { Behavior }\end{array}$} & \multicolumn{7}{c}{ Arrival Rates } \\
& 0.30 & 0.35 & 0.40 & 0.45 & 0.50 & 0.55 & 0.60 \\
\hline Pco $=1$ & 4322 & $\mathbf{4 4 7 5}$ & $\mathbf{4 5 1 2}$ & $\mathbf{4 5 5 2}$ & $\mathbf{4 5 8 2}$ & $\mathbf{4 5 8 9}$ & $\mathbf{4 6 2 0}$ \\
Pmo $=1$ & 4320 & 5038 & 5764 & $\mathbf{6 0 1 2}$ & $\mathbf{6 0 5 3}$ & $\mathbf{6 0 9 4}$ & $\mathbf{6 1 1 2}$ \\
Pur $=1$ & 4319 & 5061 & 5768 & 6345 & $\mathbf{6 3 9 8}$ & $\mathbf{6 4 3 4}$ & $\mathbf{6 4 9 4}$ \\
Pra $=1$ & $\mathbf{8 3}$ & $\mathbf{9 5}$ & $\mathbf{6 2}$ & $\mathbf{1 9}$ & $\mathbf{2 6}$ & $\mathbf{1 9}$ & $\mathbf{3 3}$ \\
\hline
\end{tabular}


Thus, as for single-lane roundabouts in Ref. 6, collective conservative behavior decreases throughput. In contrast, collective radical behavior can cause congestion on the roundabout and decrease in throughput compared to collective moderate behavior. Certainly, a distribution of driver behavior is more appropriate, but our results do reproduce for example the phenomenon of congestion on a two-lane roundabout, due to too many drivers not observing the give-way rules.

\section{Summary}

In this paper, we proposed Multi-stream Minimum Acceptable Space (MMAS) Cellular Automata (CA) models to investigate traffic flow at multi-lane roundabouts. Descriptions of MMAS models to simulate two- and three-lane roundabouts have been presented earlier. Features such as Position Delay Time and Give Way Rate are also proposed to reflect the operation of real-world roundabouts.

Two lane-allocation patterns (Patterns A and B), of two-lane roundabouts were considered, namely:

- Left-turning (LT) vehicles use left lane only, straight-through (ST) and rightturning (RT) vehicles use right lane only, i.e., only LT vehicles use the outer lane of a roundabout (Pattern A)

- LT vehicles use left lane only, RT vehicles using right lane only and ST vehicles can use both lanes (Pattern B)

Two important findings from this research are:

- Compared to Pattern A, Pattern B has better operational performance with higher throughput

- The operational performance of three-lane roundabouts is approximately the same as Pattern B two-lane roundabouts. The advantage of three-lane roundabouts is not obvious for situations where LT vehicles are filtered out directly

Other findings are as follows. For both lane allocation patterns (Patterns A and B), throughput increases linearly with arrival rate when no entrance road is in a saturated situation. It reaches a maximum when arrival rates reach their maximum for Pattern A and when arrival rate equals a critical value on one or more roads for Pattern B. When the arrival rate is larger than the critical value, saturation occurs on one or more roads. Critical arrival rates (CAR) also depend on arrival rates of other roads and turning rates for all roads.

The operational performance of a roundabout is improved when arrival rates are balanced (i.e., $A R_{1}=A R_{2}=A R_{3}=A R_{4}$ ). Throughput decreases as RT rate increases when one or more roads are saturated, as vehicles, on average, need to travel longer distances on the roundabout.

Driver behavior has an impact on the overall performance of the roundabout and individual roads. Moderate, urgent and conservative behavior leads to free-flow on the roundabout for all arrival/turning rates considered, whereas reckless behavior 
can lead rapidly to congestion for both Patterns A and B. For Pattern B, there is some difference between rational and urgent behavior in respect of throughput. Conservative behavior leads to decreased throughput for both Patterns.

Compared to Pattern A, Pattern B has better operational performance with higher throughput when all arrival rates $<0.30$. In particular, when $0.45>$ all arrival rates $>0.30$, saturation occurs for Pattern A, but not for Pattern B.

\section{Acknowledgments}

The support of the Massey University Research Fund (2004) and the ASIA 2000 Foundation Higher Education Exchange Program (2005) is gratefully acknowledged.

\section{References}

1. Z. Tian, M. Vandehey, B. M. Robinson, W. Kittelson, M. Kyte, R. J. Troutbeck, W. Brilon and N. Wu, Trans. Res. A 33, 187 (1999).

2. K. Nagel and M. Schreckenberg, J. Phys. I. 2, 2221 (1992).

3. B. Chopard, A. Dupuis and P. A. Luthi, Traffic and Granular Flow'97, eds. D. E. Wolf and M. Schreckenberg (World Scientific, Singapore, 1998), p. 153.

4. J. Wahle, L. Neubert, J. Esser and M. Schreckenberg, Parallel Comput. 27, 719 (2001).

5. H. J. Ruskin and R. Wang, Lecture Notes in Computer Science (LNCS) 2329, 381 (2002).

6. R. Wang and H. J. Ruskin, Comput. Phys. Commun. 147, 570 (2002).

7. O. Biham, A. A. Middleton and D. Levine, Phys. Rev. A 46, R6124 (1992).

8. D. Chowdhury, L. Santen and A. Schadschneider, Phys. Report 329, 199 (2000).

9. R. Jiang, Q. S. Wu and H. B. Wang, Phys. Rev. E 66, 036104 (2002); 67, 068102 (2003).

10. P. Simon and H. A. Gutowitz, Phys. Rev. E 57, 2441 (1998).

11. H. W. Lee, V. Popkov and D. Kim, J. Phys. A 30, 8497 (1997).

12. R. Wang, PhD thesis, Dublin City University, Ireland (2003).

13. M. Fukui and Y. Ishibashi, J. Phys. Soc. Jap. 66, 514 (1997).

14. J. Freund and T. Pvschel, Physica A 219 (1995).

15. B. Chopard, P. A. Luthi and P. A. Queloz, J. Phys. A 29, 2325 (1996).

16. J. Esser and M. Schreckenberg, Int. J. Mod. Phys. C 8, 1025 (1997).

17. K. Nagel, Traffic and Granular Flow'95, eds. D. E. Wolf and M. Schreckenberg (World Scientific, Singapore, 1996), p. 41.

18. M. R. Liu, personal communication (2005).

19. M. Kyte, Z. Tian, Z. Mir, W. Harneedmansoor, M. Kittelson, M. Vandehey, B. Robinson, W. Brilon, L. Bondzio, N. Wu and R. J. Troutbeck, Final Report: Vol. 1 Two-Way Stop-Controlled Intersections, National Cooperative Highway Research Program, Project 3-46 (1996), accessed 28 August 2005, http://www.nap.edu/books/nch005/html/.

20. R. J. Troutbeck and W. Brilon, Unsignalized Intersection Theory, Traffic Flow Theory, TRB (1997), accessed 28 August 2005, http://www.tfhrc.gov/its/tft/chap8.pdf.

21. W. Plank and E. A. Catchpole, Traffic Engineering and Control 25, 327 (1984).

22. G. Bottom and R. Ashworth, Ergonomics 21, 721 (1978).

23. J. Miller, Theory of Traffic Flow and Transportation - Proc. Berkeley California Int. Symp., ed. G. Newell (Elsevier, 1972), p. 215. 
24. R. J. Troutbeck, Research Report, Vol. 92-1, Queensland University of Technology (1992).

25. W. Brilon, R. Kening and R. J. Troutbeck, Intersections without Traffic Signals Proc. Portland Oregon Int. Symp., ed. M. Kyte, University of Idaho (1997).

26. O. Hagring, Trans. Res. B 34, 293 (2000).

27. R. J. Troutbeck, Proc. 12th Australian Road Research Board Conference, Vol. 12 (1985), p. 62.

28. Z. Tian, R. J. Troutbeck, M. Kyte, W. Brilon, M. Vandehey, W. Kittelson and B. M. Robinson, Highway Capacity - Proc. 4th Int. Symp. (2000), p. 397.

29. D. W. Harwood, J. M. Mason and E. B. Robert, Trans. Res. A 33, 199 (1999).

30. R. J. Troutbeck and S. Kako, Trans. Res. A 33, 291 (1999).

31. M. Tracz and S. Gondek, Highway Capacity - Proc. 4th Int. Symp. (2000), p. 471.

32. W. Robinson, Z. Tian, W. Kittelson, M. Vandehey, M. Kyte, N. Wu, W. Brilon and R. J. Troutbeck, Trans. Res. A 33, 217 (1999).

33. R. J. Troutbeck, Proc. 15th Australian Road Research Board Conference, Vol. 15, Part 5 (1990).

34. R. J. Troutbeck and S. Kako, Trans. Res. A 33, 291 (1999).

35. J. Golias, Gap Acceptance Delay and Capacity for Vehicles Crossing a Priority Stream, Transport Studies Group, University College, London (1981).

36. M. R. C. McDowell, J. Wennell, P. A. Storr and J. Darzentas, Special Report, Vol. 776, Transport and Road Research Laboratory, Crowthorne, England (1983).

37. E. A. Catchpole and A. W. Plank, Transportation Research 20, 6 (1986).

38. B. Jia, R. Jiang and Q. S. Wu, Int. J. Mod. Phys. C 15, 381 (2004).

39. R. Wang and H. J. Ruskin, LNCS 2667, 577 (2003).

40. S. Teply, M. I. Abou-Henaldy and J. D. Hunt, Traffic Engineering and Control 38, 9 (1987).

41. Y. Nishida, JSAE Review 20, 375 (1999).

42. G. J. Wide, Risk Anal. 2 (1982). 